\title{
Special issue on swarm intelligence and its applications to engineering
}

\author{
Jagdish Chand Bansal $^{1} \cdot$ Harish Sharma ${ }^{2} \cdot$ Kusum Deep $^{3} \cdot$ Kedar Nath Das $^{4}$. \\ Atulya Nagar ${ }^{5}$
}

Published online: 31 July 2018

(C) The Society for Reliability Engineering, Quality and Operations Management (SREQOM), India and The Division of Operation and Maintenance, Lulea University of Technology, Sweden 2018

This special issue of the International Journal of System Assurance Engineering and Management (IJSA) is an excellent collection of research articles in the field of swarm intelligence and its applications to engineering. An open Call for Paper was issued for this special issue. The guest editors feel happy to produce this special issue of one of the most reputed journal IJSA.

From a wide range of interesting research papers on various aspects of swarm intelligence, the editors carefully selected 19 papers through rigorous peer-review process. The final decision for the inclusion of 19 papers has been strictly based on the outcome of the rigorous peer-review process. A brief summary of these is given below.

In the first article, Assad et al. hybridized Harmony Search and Hill Climbing algorithm and applied to solve the Sudoku problem. Chaudhari et al. incorporated

Jagdish Chand Bansal

jcbansal@gmail.com

Harish Sharma

hsharma@rtu.ac.in

Kusum Deep

kusumdeep@gmail.com

Kedar Nath Das

kedar.iitr@gmail.com

Atulya Nagar

nagara@hope.ac.uk

South Asian University, New Delhi, Delhi, India

2 Rajasthan Technical University, Kota, India

3 Indian Institute of Technology, Roorkee, India

4 National Institute of Technology, Silchar, India

5 Liverpool Hope University, Liverpool, UK 'swapping crossover' and 'cut \& fill' operators to Troop Search Optimization (TSO) algorithm to make it more robust. Then Artificial bee colony algorithm inspired fitness-based solution search process is incorporated with the PSO solution search process by Kumar et al. In the next article, Kumar et al. proposed a new ACO algorithm (ACO-LD), which incorporates Laplace distribution based interaction scheme among the ants. In order to avoid the problem of stagnation, an additional diversification mechanism is also introduced. A variant of ABC, namely Selfadaptive Position update in $\mathrm{ABC}$ (SPABC), in which three position update strategies are incorporated in employed bee phase based on the fitness of the solutions is proposed by Jadon et al. In another research, Rehani et al. proposed the multi-objective NSGA-II based scheduling algorithm for workflow applications. Three conflicting criterion optimized simultaneously: execution time, reliability and energy consumption for executing the workflow application in cloud environment. A novel clustering method based on Biogeography based optimization is proposed to extend the capabilities of traditional clustering methods by Pandey et al. while Poonia applied artificial bee colony algorithm and AntNet to solve the issue in Vehicular adhoc network (VANETs) like data aging, heavy cost and message prioritization. Pandey et al. presented a new approach based on discrete glowworm swarm optimization algorithm for solving the 3-dimensional path planning problem for a flying vehicle. The flying vehicle's task is to generate a viable trajectory for a source point to the destination point keeping a safe distance from the obstacles present in the way. Chaotic flower pollination algorithm (CFPA) proposed by Kaur et al. Then a novel variant of bat algorithm based on dynamic frequency is proposed by Tripathi et al. The proposed variant is also hybridized with 
K-means algorithm to present a new approach for clustering in distributed environment. Bharill et al. proposed an interesting hybrid fuzzy clustering approach referred as quantum-inspired evolutionary fuzzy c-means (FCM) algorithm. The authors integrated the concept of quantum computing with FCM to evolve the fuzziness factor $m$ in several generations. Agarwal et al. reviewed various variants of particle swarm optimization (PSO) and proposed a variant of PSO, namely map reduce two phases quantum behaved fuzzy rule PSO (MR-TP-QFPSO). Kaur et al. proposed an interesting algorithm, which is, based on hybridization of K-Means and Firefly Algorithm for anomaly detection. Prakash et al. modified artificial bee colony $(\mathrm{ABC})$ algorithm in two ways to better balance the exploration and exploitation capabilities. Authors introduced gbest-guided search mechanism and a crossover operator in ABC. Then Agrawal et al. reviewed a recent swarm intelligence algorithm, spider monkey optimization. Jain et al. presented automated cryptanalysis of the reduced multiplicative knapsack cryptosystem using three nature inspired algorithms, cuckoo search, particle swarm optimization and genetic algorithm. Rout et al. employed an approach to construct the remote control input signal from locally measurable quantity so that the control system performs satisfactorily under communication failure. Hybridization of differential evolution and pattern search is employed for static synchronous series compensator-based damping controller design in Single Machine Infinite Bus and multi-machine power systems. Jain et al. proposed discrete cuckoo search optimization algorithm. The authors also applied the developed algorithm to solve a transposition cipher.

The main aim of this special issue has been to make available recent research in the field of swarm intelligence. We much hope that many students, researchers, and academics will be benefitted with this publication.

As guest editors, we would like to express our deep thanks to the Editors-in-Chief, Prof. A. K. Verma, Prof. P.K. Kapur and Prof. U. Kumar, for providing us with the opportunity to host this special issue in IJSA. We also thank the authors for their contributions, including those whose papers were not included. We are also thankful to reviewers whose constructive and intensive comments made these papers publishable. Last but not least, we express our sincere gratitude for the editorial staff of publisher Springer Nature for the support and producing this collection of research. 